إتقان اللفات الأجنبية كأسلوب لتحسين الاداء الأكاديمي لطلبة المحاسبة

- سراسة ميدانية لطلبة المحاسبة بجامعة جيجل-

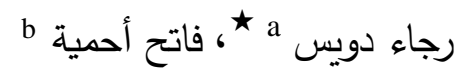

• rajadouis@ @ gmail.com .a

· Fateh742002@yahoo.fr.b

Received date: 30/ 05/20**, Accepted date: 20/ 06/2021, online publication date: 30/ 06/2021

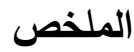

تسعى هذه الدراسة إلى إبراز أثر اتقان اللغات الأجنبية على الأداء الأكاديهي لطلبة الدحاسبة وذلك في إطار إيجاد أساليب من شانها تحسين جودة التعليم الدحاسبي في الجزائر وتدويله، وللخروج بنتائج للدراسة تم إتباع المنهجين الوصفي، التحليلي وتوزبع الاستبيان على 30

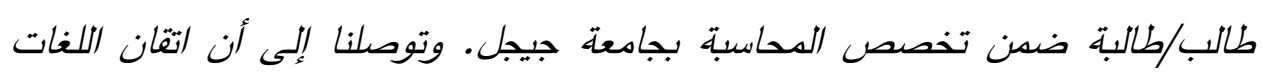

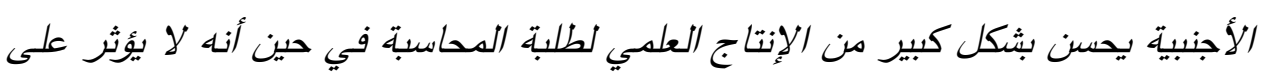
تحصيلهم العلمي واندماجهم المهني في البيئة الجزائرية. الكلمات الدالة: لغات أجنبية؛ أداء أكاديمي؛ تعليم محاسبي؛ انتاج علمي. تصنيفات (JEL):

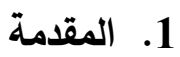

يعتبر موضوع الأداء الأكاديمي لطلبة المحاسبة من أكثر الموضوعات التي تثار في الساحة

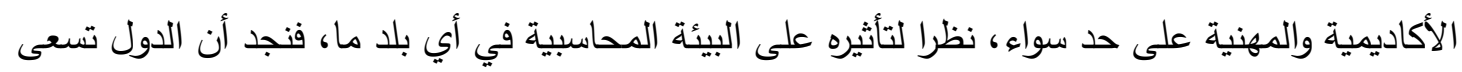
جاهدة إلى ابتكار أسس وأساليب وطرق متتوعة بغية الارتقاء بأداء طلبتها فمنها من يستهدف المناهج التعليمية ويسعى إلى تحديثها وتقريبها من الممارسات المهنية المحاسبية، ومنها من يركز على ترقية الهيئة التدريسية وتدريبها، ومنها من يستهدف الطالب في حد ذاته. 
إتقان اللغات الأجنبية كأسلوب لتحسين الاداء الأكاديمي لطلبة المحاسبة

يمثل الطالب أصدق مقياس للحكم على نجاعة التعليم المحاسبي، لأنه يعتبر مخرجات العملية التعليمية، فأداء الطالب يعبر عنه من خلال العديد من المؤشرات مثل تحصيله العلمي، إنتاجه العلمي ودرجة اندماجه في الوسط المهني... ، وجميع هذه المؤشرات لا تتأثر فقط بهيئة التدريس أو المناهج التعليمية خاصة مع ظهور النهج التعليمي القائم على البحث، بحيث أننا في عصر العولمة والتكنولوجيا لذا يمكن الحصول على كم هائل من المعرفة من جميع أنحاء العالم، ولكي نحصل على طلاب مؤهلين عالميًا يجب أن يتم استقطاب العلوم من جميع أنحاء العالم، لكن يبقى العائق الوحيد أمام هذا هو الجسر هن اللغوي، فاللغة هي الأداة الرئيسية للتواصل واكتساب المعرفة.

اللغات الأجنبية تعتبر المفتاح الرئيسي في النشاط الأكاديمي والمهني على حد سواء ،فمن خلالها يمكن تواصل و اكتساب معارف وثقافات وخبرات متتوعة من العديد من دول العالم، بدل الاقتصار على الى المعرفة المحلية خاصة في علم المحاسبة الذي يتطور باستمرار ، فنجد أن الطلب مرتفع عليها، إذ أن معظم دول العالم تخلت عن لغاتها المحلية في التعليم العالي و أدرجت العديد من اللغات الأجنبية الرائدة كلغات تدري أساسية لمناهجها ،وكذلك نجد أن معظم الشركات تستتد في التوظيف على معيار امتلاك الفرد لخزان اللغات الأجنبية للحكم على كفاءته. إثكالية البحث: ومن خلال ما سبق تدفعنا المعلومات المطروحة سابقا إلى طرح التساؤل الرئيسي لهذه الورقة البحثية كما يلي: "هل يؤثر إتقان اللغات الأجنبية على الاداء الأكاديمي لطلبة المحاسبة في الجزائر ؟"

الأسئلة الفرعية: يتفرع من السؤال الرئيسي الأسئلة الفرعية التالية:

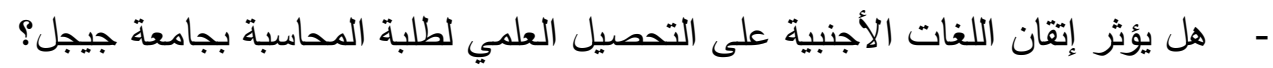

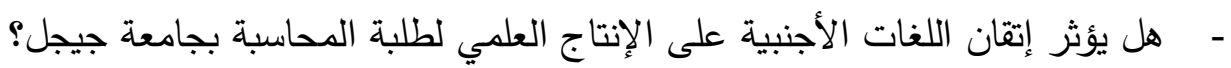
- هل يؤثر إتقان اللغات الأجنبية على الاندماج المهني لطلبة المحاسبة بجامعة جيجل؟ الإنج

الفرضيات: سوف نحاول الإجابة على الأسئلة الفرعية السابق ذكرها بواسطة الفرضيات المؤقتة التالية: - - يؤدي إتقان اللغات الأجنبية إلى تحسين التحصيل العلمي لطلبة المحاسبة بجامعة جيجل. - - ميؤدي إتقان اللغات الأجنبية إلى تحسين الإنتاج العلمي لطلبة المحاسبة بجامعة جيجل. - - يؤدي إتقان اللغات الأجنبية إلى تحسين التحصيل العلمي لطلبة المحاسبة بجامعة جيجل. أهمية البحث: نحاول من خلال هذه الورقة البحثية تسليط الضوه على أهم الجوانب المساعدة في تعزيز كفاءة طلبة المحاسبة وتعزيز جودة التعليم المحاسبي بالجزائر والارتقاء به وتدويله وتسهيل العلمية التعليمية لمالب وصقل معرفته. أهداف البحث: يمكن حصر أهدف الورقة البحثية التالية فيما يلي: - التعرف على مستوى إتقان اللغات الأجنبية لدى طلبة المحاسبة. - - التعرف على أثار إتقان اللغات الأجنبية على أداء طلبة المحاسبة التعان - - محاولة إيجاد أساليب من شانها الارتقاء بجودة التعليم المحاسبي في الجزائر . 
المجلا. 5، العدد. 1 (جوان 2021)؛ 565N: 2602-565

منهج الدراسة: تم اعتماد المنهج الوصفي في الجانب النظري لهذه الورقة البحثية وعلى منهج تجريبي من خلال القيام بدراسة ميدانية تم فيها توزيع استبيان على طلبة المحاسبة بجامعة جيجل وتحليله إحصائيا. محاور البحث: تم تقسيم هذه الورقة البحثية إلى أربعة محاور، بحيث يتطرق المحور الأول إلى مفاهيم حول إتقان اللغات الأجنبية، والمحور الثاني يتطرق إلى الاداء الأكاديمي المحاسبي، في حين يتطرق إلى لإنه

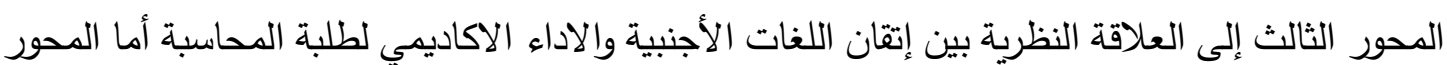
الرابع والأخير يتطرق إلى الدراسة الميدانية لطلبة المحاسبة بجامعة جيجل. 2. 2 مراجعة الأدبيات

1- 1 - 1 مفاهيم حول إتقان اللغات الأجنبية إن موضوع إتقان اللغات أصبح من ضروريات العولمة الحالية فهناك معايير محددة تمكننا من تحديد ما إذا كان المتعلم يتقن لغة معينة أم لا.

1-1 يوجد العديد من التعريفات لهذا المصطلح وفيما يلي سنعرض التعريفات التالية التي تتوافق مع أغراض

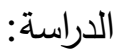
- مفهوم أساسي يشير إلى قدرة المتكلم على فهم وترجمة وإنتاج عدد لا متتاهي من الكلمات والجمل (حليمة، 2009). - من المعترف به عمومًا أن مفهوم الكفاءة في لغة ثانية أو لغة أجنبية يشمل جوانب القدرة على فعل شيء ما مع اللغة ("معرفة كيف") بالإضافة إلى معرفتها ("معرفة ماذا") . Harsch, 2017, pp) .250-253) - حسب (Ruiz and Dunn Blagojevich)، فان متعلمو اللغة الإنجليزية يتواصلون مع المعلومات والأفكار والمفاهيم اللازمة للنجاح الأكاديمي في مجال الدراسات الاجتماعية. من خلال التعريفات السابقة نصيخ التعريف التالي: الكفاءة اللغوية تتعلق بقدرة الفرد على التكلم، استماع، وفهم، وقراءة لغة معينة واستخدامها لهدف معين. 2-1 أنواع الكفاءة اللغوية وفية حسب (Cummins) يمكن التمييز بين نوعين أساسين من الكفاءة اللغوية: - كفاءة الاتصال الأساسية بين الأشخاص: ويعرف اختصارا ب (BICS) وهي المهارات اللازمة للتفاعل مع أشخاص آخرين في مواقف الحياة اليومية. - الكفاءة اللغوية الأكاديمية المعرفية: تعرف اختصارا ب (CALP)تم تعريفها على أنها المعرفة والمهارات الأكاديمية المكتسبة في التعليم الرسمي، وبالتالي يثير إلى نوع من الكفاءة اللازمة للمهام (Harsch, 2017). هنا نرى أنه تم ربط الكفاءة اللغوية بجانب محدد وهو الجانب الأكاديمي. تعتبر الكفاءة اللغوية في لغة أجنبية معينة أقصى ما يمكن للمتعلم إتقانه والوصول إليه، ولكن يمكن للفرد أن يتقن لغة معينة في مجال معين فقط مثال ذلك هو وجود شخص ما يتقن انجليزية محاسبية 
إتقان اللغات الأجنبية كأسلوب لتحسين الاداء الأكاديمي لطلبة المحاسبة

فقط، أو فرنسية مالية فقط، اسبانية فندقية ...الخ، وهو ما يعرف بلغة التخصص أو باللغة لأغراض خاصة (Language for Specific Purposes) ويشار إليها اختصار ب.(LSP)

3-1 تعريف اللغة لأغراض خاصة

نختصر على التعريفات التالية:

- حسب (Strevens) فان اللغة لأغراض خاصة مصممة لتلبية احتياجات محددة للمتعلم، وذات صلة بتخصصات، مهن، وأنشطة معينة. فهي تتمحور حول اللغة المناسبة لتلك الأنشطة في النحو والمفردات والخطاب والدلالات بعبارة أخرى تدمج اللغة لأغراض التخصص كل من علم اللغة والمعرفة الخاصة بمجال معين بناء على احتياجات المتعلمين.(Trace, 2015) - مفهوم يقصد منه الملامح التي تتميز بها اللغة في استعمالات المتخصصين في حقول المعرفة المختلفة (كالعلوم البحت مثل الفيزياء أو الكيمياء أو الرياضيات والعلوم التطبيقية كالهندسة مثلا) أو الممارسين لمهن خاصة (كالصحفيين ورجال القانون والأطباء وغيرهم) (صالح، 2013). - تعليم اللغة لأغراض خاصة، مدخل لتعليم اللغة تستتد كافة عناصره، من أهداف ومحتوى وطريقة تدريس، إلى الأسباب التي دفعت الدارسين لتعلم اللغة (محمد، 2019). من خلال ما سبق يظهر بأن اللغة لأغراض خاصة فرع من علم اللغة أين يتقن فيه المتعلم مجال محدد من لغة أجنبية لغرض الاستفادة منه في أبحاثه الأكاديمية أو في مهنته. 1-4 أنواع اللغة لأغراض خاصة الهن يستخدم هذا المدخل تقرببا في جميع لغات العالم ولكن أشهرها هي اللغة الانجليزية والفرنسية لأغراض

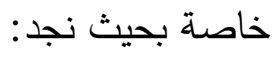

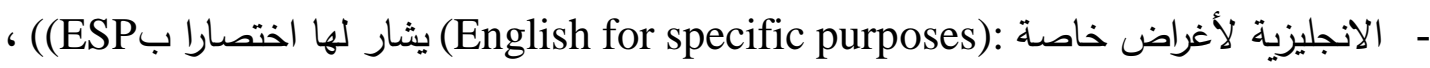
ونعني بها تدريس اللغة الإنجليزية المستخدمة في الدراسات الأكاديمية أو تدريس اللغة الإنجليزية للأغراض

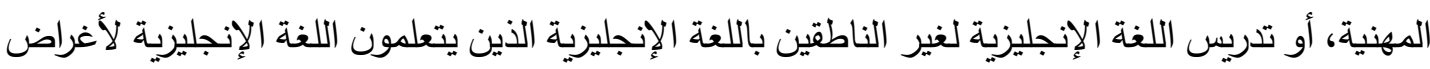

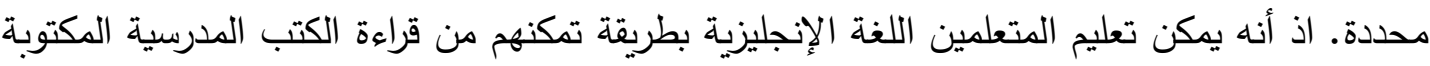
باللغة الإنجليزية ذات الصلة بمجال تخصصهم، أو استخدام اللغة الإنجليزية في حياتهم المهنية المستقبلية، فهي تسعى إلى تلبية الاحتياجات اللغوية للمتعمين الذين يحتاجون إلى اللغة الإنجليزية من أجل القيام بأدوار محددة (مثل الطالب والمهندس والممرض.(Agustina, 2014) - يشار لها اختصارا (français sur objectifs spécifiques): ب (FOS)وهي منهجية تستهدف جمهورًا مهنيا وفقًا للطلب لتجسيد التواصل في مواقف دقيقة ومحددة في مجال أو قطاع مثل السياحة (في الفنادق والمطاعم) وما إلى ذلك. وبالتالي، تكون قادرًا على الرد على العملاء الناطقين بالفرنسية. على سبيل المثال، لدينا سياحة في جزيرة فوغو، والجمهور المحترف

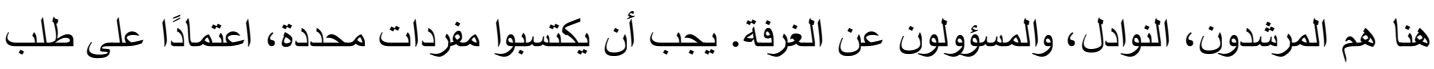

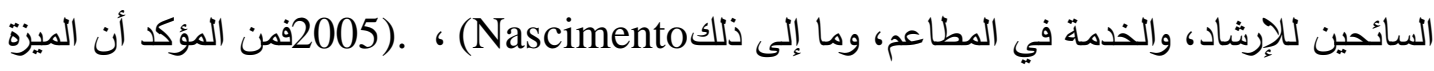


المجلا. 5، العدد. 1 (جوان 2021)؛ ISSN: 2602-5655)

الرئيسية لـ (FOS) هي جمهورها. هؤلاء غالبًا ما يكونون محترفين أو أكاديميين يرغبون في أخذ دورات اللغة الفرنسية لأغراض مهنية أو جامعية(ferrari, 2016) .

2- الأداء الأكاديمي المحاسبي

يعتبر الأداء الأكاديمي انعكاس لنتائج التعليم، كما أن تحقيق التميز الأكاديمي للطلاب يعتبر الدافع الأول للمؤسسات الأكاديمية . 2-1 تعريف الأداء الأكاديمي المحاسبي لا يوجد تعريف دقيق لهذا المصطلح بحد ذاته ولكن هناك بعض الاجتهادات من طرف الباحثين لإيجاد

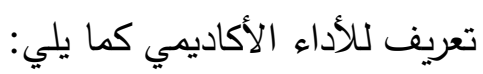
- المعرفة المكتسبة من قبل الطالب والتي يتم تقييمها بالعلامات من طرف الأستاذ و/أو الأهداف (Saumya Kumar, التعليمة التي وضعها الطلاب والأساتذة ليتم تحقيقها خلال فترة زمنية محددة 2021).

يمكن تعريف الاداء الأكاديمي من ناحية اخرى على انه اكتساب المعرفة، المهارات، الكفاءة، أعلى (Saumya Kumar, الدرجات، وانجازات اكاديمية، وتامين حياة مهنية، والنية والمثابرة نحو التعليم 2021).

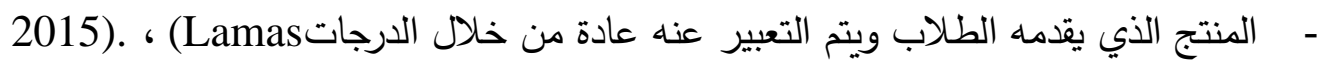
- مما سبق يمكن استخراج التعريف التالي للأداء الأكاديمي المحاسبي: عبارة عن الانجازات الاكاديمية المحاسبية التي تشمل المعرفة والمهارات المحاسبية التي يكتسبها الطالب ويتم تقييمها من طرف المؤسسة التعليمية. 2-2 العوامل المؤثرة على الأداء الأكاديمي المحاسبي التعليه أثبتت مجموعة من الأبحاث أن الاداء الأكاديمي بصفة عامة يختلف بتغير العديد من العناصر نذكر منها:

- - العوامل الثخصية: مثل الجنس، العمر، الثخصية، الاهتمام. - أساليب التعلم، مهارات التعلم، القدرات، عادات الدراسة، التحفيز . - - الانجازات الأكاديمية السابقة. - العوامل النفسية: الجاهزية العقلية، نمط التفكير، عملية التفكير، الموقف النفسي. - العوامل البيئية: مثل الجو في المنزل، الجو في المؤسسة التعليمية. - العوامل الاقتصادية: الوضع الاقتصادي للطلاب.

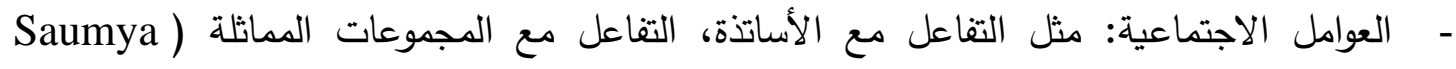
.(2021،Kumar 2-3 قياس الأداء الأكاديمي المحاسبي نظرا للطبيعة الغير متبلورة لهذا المصطلح، فان الباحثين استخدموا العديد من الأدوات لقياسه، وسنتطرق إلى العناصر المحددة في الثكل التالي والمستوحاة من التعريفات المختلفة لهذا المصطلح: 
شكل 1: بعض معايير قياس الأداء الأكاديمي

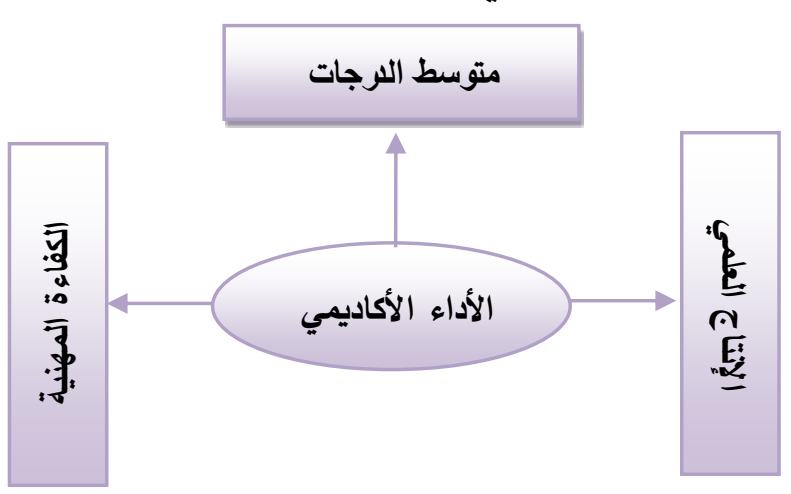

المصدر :تم اعداده بالاعتماد على (Tachibana، 2017)، و) و Saummed Abubakar, 2018، Kumar)

\section{3- إتقان اللغات الأجنبية والأداء الأكاديمي لطلبة المحاسبة}

تظهر العلاقة والتأثير بين اتقان اللغات الأجنبية والأداء الأكاديمي لطلبة المحاسبة كما يلي: حسب نظرية الذكاء المتعدد التي جاء بها (Howard Gardner) ، فان هناك 7 وسائل تمكن الناس من فهم وإدراك العالم وحل المشاكل، ويعتبر الذكاء اللغوي واحدا من هذه الوسائل ونقصد به القدرة على تعلم اللغات واستخدامها لتحقيق أهداف معينة (Abdirahman M. Addow), 2013 ، بحيث أن اللغة قناة لنقل وتداول المعرفة بصفة عامة وتسمح بالتبادل العلمي والثقافي بين الدول مما يسهل العملية التعليمية، خاصة مع ظهور النهج التعليمي القائم على البحث (RBL) والذي انتشر بثكل كبير في الجامعات من خلد البحوث التي تقدم للطلبة كمنهج للتدريس(M S Sumbawati، 2018) فيحتاج الطالب لأداة اللغة لمساعدته في هذا لمجال .كما نصت كل من وزارة التعليم البريطانية سنة 1992 والمجلس الأعلى لتقييم

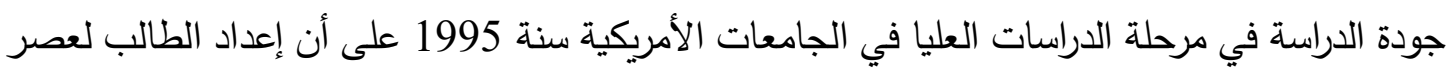
العولمة من خلال تعلم لغة أجنبية يعتبر كمؤشر لتقييم لجودة الخدمة التعليمية في المؤسسة التعليمية (زكري، 2013)، إذ أن إتقان اللغات الأجنبية تمثل أحد الأوجه المساعدة بشكل غير مباشر في الرفع من اداء الطلبة، و بالتالي تعزيز جودة التعليم المحاسبي وذلك باعتبار أن جودة التعليم المحاسبي تعتمد على إلى التحيز جودة أداء طلاب المحاسبة (ليبيا رقم براءة الاختراع 866887، 2009) وذلك من خلال العناصر التالية: 3-1 تحسين جودة التحصيل العلمي للطالب يعتبر الطالب العنصر المهم في التعليم المحاسبي، وإتقانه للغات الأجنبية يمكن أن يساعد في الرفع من تحصيله العلمي، على سبيل المثال يسمح إتقان اللغة الانجليزية أو على الأقل إتقان اللغة الانجليزية

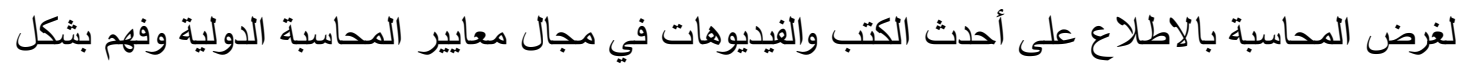
أعمق و أفضل لمعايير الدولية للتقارير المالية ذلك لأن الطالب يتلقاها من مصادر عديدة و من المنبع دون تحريف أو تأويل وبشكل أسرع وأسهل وأكثر كفاءة بدل انتظار ترجمتها أو تلقيها من كتب بلغة المحلية التي تعتبر محدودة وضيقة، والتي يمكن أن لا تتعرض لمواضيع يحتاجها الطالب مما يؤدي إلى 
المجلا. 5، العدد. 1 (جوان 2021)؛ 565N: 2602-565

رفع التحصيل العلمي في مقاييس المحاسبة عوضا عن الاعتماد على اللغة المحلية فقط ـ فستمنح اللغة الإنجليزية الطالب قدرًا غير محدود من المعرفة (Aysha Khaled Almajed، 2015) 3-2 تحسين الإنتاج العلمي للطالب ومن جهة أخرى يساعد إتقان اللغات الأجنبية على الاطلاع على حصيلة اكبر من الإنتاج العلمي الدولي خاصة اللغة الانجليزية فأعظم الأدب في العالم مكتوب باللغة الإنجليزية، ذلك ان معظم الطلاب يقل اهتمامهم بقراءة المقالات الانجليزية بسبب المهارات اللغوية المحدودة (M S Sumbawati, 2018) ولكي يتمكن الطالب من الاستمتاع بها يجب أن يتعلم اللغة الإنجليزية (Aysha Khaled Almajed، يعرةه 2015)، وبالتالي يساعد إتقان اللغات على تسهيل الإنتاج العلمي للطالب وتدريبه، ومنحه فرصة حضور المؤتمرات والفعاليات الدولية والحصول على المزيد من الثقافات(Aysha Khaled Almajed، 2015) وإعطائه إمكانية لنشر مقالات وبحوث أكاديمية محكمة، وهذا ما دفع بالدول المتقدمة والنامية على حد سواء إلى تدريس المناهج المحاسبية بلغة غير اللغة المحلية وغالبا ما تكون الانجليزية.

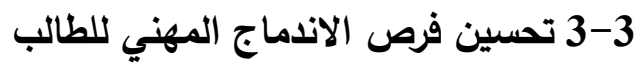

يمكن إتقان اللغات الأجنبية الطالب من فهم مصطلحات العالم المهني بسرعة وتسهيل اندماجه في بيئة الأعمال وبالتالي الرفع من فرص تثغيله عند التخرج ذلك أنه دائما ما تكون اللغة المستخدمة في الجانب المهني وأماكن العمل غير محلية وغالبا ما تكون اللغة الفرنسية أو الانجليزية، وبالنسبة لبيئة الأعمال الجزائرية غالبا ما يتم استخدام اللغة الفرنسية في المسك المحاسبي. كذلك بالنسبة للغة الانجليزية يسمح التحدث بها للطالب بالتواصل بشكل فعال في العديد من البلدان وهذا سيجعل الطالب قادرًا على اقتناص فرص جديدة للعمل في بلد أجنبي، حيث سيكون الطالب مرغوبًا فيه بثكل أكبر وفي وضع أقوى للتقدم لوظيفة في الخارج (Aysha Khaled Almajed, 2015).

3- البيانات ومنهجية الدراسة فيما يلي سنتعرض لمنهجية الدراسة والعينة والنتائج ومناقتتها.

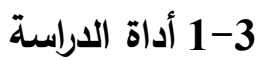
تم الاعتماد على الاستبيان الالكتروني للإجابة على أسئلة الدراسة فتم تقسيمه الى محورين، الأول: يقيس

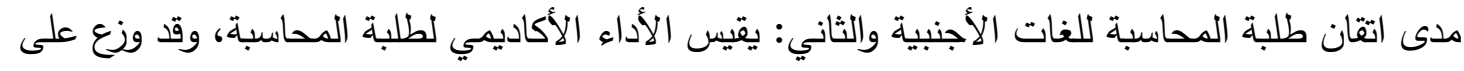
30 طالب/طالبة ينتمون الى جامعة محمد الصديق بن يحي -جيجل.3-3 عينة الاراسة كما هو مبين في الثكل 2 نلاحظ أن أغلبية العينة كانت مكونة من طالبات وبدرجة أقل طلاب. 
شكل 2: توزيع العينة حسب الجنس

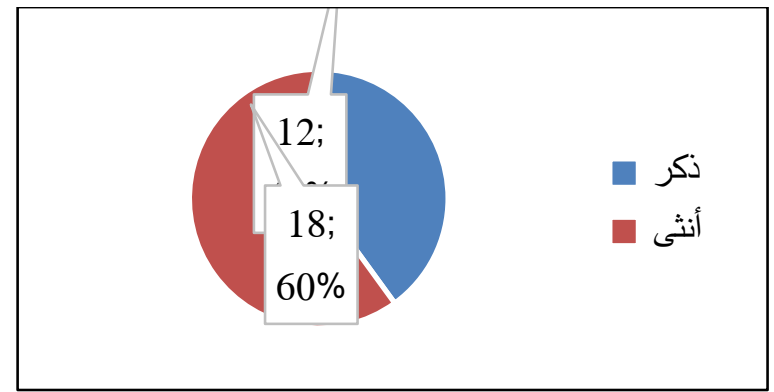

Excel المصدر : مخرجات برنامج وكذا مستوى الماستر هو السائد على العينة حسب الثكل 3 وبدرجة أقل دكتوراه تليها ليسانس. شكل 3: توزيع العينة حسب المستوى العلمي

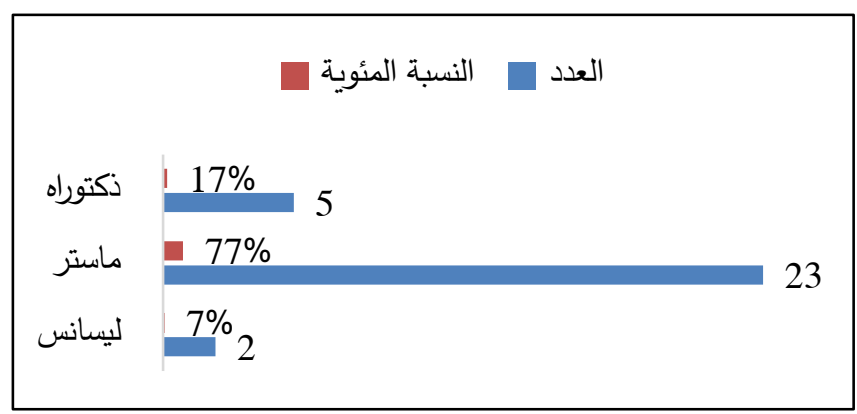

المصدر : مخرجات برنامج Spss 4- عرض نتائج الدراسة

سنقوم بتحليل محاور الاستبيان لغرض الإجابة على أسئلة الدراسة. نتائج المحور الأول: يظهر من خلال الجدول 1 أسفله بشكل عام أن مستوى اتقان اللغات الأجنبية لاى الاى الته طلبة المحاسبة ضعيف وهو ما يثير اليه المتوسط العام للمحور ، بحيث أظهرت النتائج أن مستوى الطلبة في اللغتين الإنجليزية والفرنسية لأغراض المحاسبة ضعيف، فمن ناحية قدرة الاستماع(العبارة او2) كان المستوى ضعيف في الإنجليزية ومتوسط في الفرنسية ومن ناحية قدرة القراءة(العبارة3 4) كذلك كان

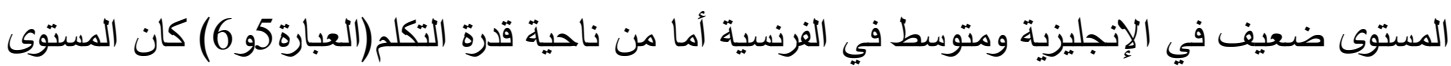
ضعيف في كلا اللغتين. يمكن ان يرجع ذلك بشكل أساسي الى أسلوب التدري المعتمد في أغلب الجامعات الجزائرية بحيث يتم تدريس تخصص المحاسبة باللغة العربية. جدول 1: نتائج المحور الأول "مدى اتقان طلبة المحاسبة للفات الأجنبية"

\begin{tabular}{|c|c|c|c|c|c|c|c|}
\hline الاتجاه & الانحراف & المتوسط & ممتاز & متوسط & ضعيف & & المحور الأول \\
\hline \multirow{2}{*}{ ضعيف } & \multirow{2}{*}{0,71840} & \multirow{2}{*}{1,6333} & 4 & 11 & 15 & عدد & \multirow{2}{*}{ 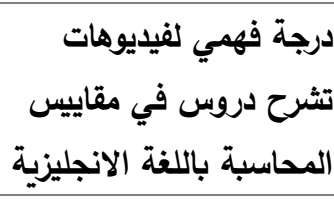 } \\
\hline & & & 13.3 & 36.7 & 50 & $\%$ & \\
\hline متوسط & 0,71438 & 1,8000 & 5 & 14 & 11 & عدد & \\
\hline
\end{tabular}


المجلد. 5، العدد. 1 (جوان 2021)؛

\begin{tabular}{|c|c|c|c|c|c|c|c|}
\hline & & & 16.7 & 46.7 & 36.7 & $\%$ & الثرحة دربة فهمي لفيديوهات \\
\hline \multirow{2}{*}{ ضعيف } & \multirow[t]{2}{*}{0,71840} & \multirow{2}{*}{1,6333} & 4 & 11 & 15 & عدد & \multirow{2}{*}{ محسبة استيعابي لكتب } \\
\hline & & & 13.3 & 36.7 & 50 & $\%$ & \\
\hline \multirow{2}{*}{ متوسط } & \multirow{2}{*}{0,65126} & \multirow{2}{*}{1,7000} & 3 & 15 & 12 & عدد & \multirow{2}{*}{ نسبة استيعابي لكتب } \\
\hline & & & 10 & 50 & 40 & $\%$ & \\
\hline \multirow{2}{*}{ ضعيف } & \multirow{2}{*}{0,62606} & \multirow{2}{*}{1,4333} & 2 & 9 & 19 & عدد & \multirow{2}{*}{ الانجليزية في المواضيع النقاش باللغة } \\
\hline & & & 6.7 & 30 & 63.3 & $\%$ & \\
\hline \multirow{2}{*}{ ضعيف } & \multirow{2}{*}{0,62881} & \multirow{2}{*}{1,5333} & 2 & 12 & 16 & عدد & \multirow{2}{*}{ المكرنسية النقاش باللغة } \\
\hline & & & 6.7 & 40 & 53.3 & $\%$ & \\
\hline \multirow{2}{*}{ ضعيف } & \multicolumn{2}{|c|}{1,6222} & \multicolumn{5}{|c|}{ المتوسط العام للمحور } \\
\hline & \multicolumn{2}{|c|}{0,53594} & \multicolumn{5}{|c|}{ الانحراف المعياري للمحور } \\
\hline
\end{tabular}

المصدر: مخرجات برنامج SPSS

نتائج المحور الثاني: يظهر من خلال الجدول 2 المبين في الأسفل بشكل عام أن مستوى تمكن طلبة المحاسبة من تخصصهم متوسط عموما وذلك من خلال المتوسط العام للمحور بحيث أن متوسط درجاتهم في المقاييس المحاسبية ممتاز (العبارة1و2و3) في حين أن انتاجهم العلمي في مجال المحاسبة باللغات الأجنبية ضعيف(العبارة4و5)،أما بالنسبة لاندماجهم المهني في مجال المحاسبة سواء من خلال التربصات الميدانية أو فرص العمل المستقبلية ممتاز (العبارة6و7)،يمكن تفسير ذلك من خلال جهود الأساتذة في تقديم المادة العلمية من ناحية تزويد الطلبة بمطبوعات وكتب من انتاج الأساتذة باللغة العربية لمساعدتهم على التمكن من المقاييس المحاسبية، وكذلك من ناحية الاندماج المهني راجع الى مساعدة عمال المؤسسات للطبة خاصة العمال ذوي الخلفية الأكاديمية الذين كانوا خريجين سابقين من الجامعة.

جدول 2: نتائج المحور الثاني "الأداء الأكاديمي لطلبة المحاسبة"

\begin{tabular}{|c|c|c|c|c|c|c|c|}
\hline الاتجاه & الانحراف & المتوسط & ممتاز & متوسط & ضعيف & & المحور الأول \\
\hline \multirow{2}{*}{ ممتاز } & \multirow{2}{*}{0,50401} & \multirow{2}{*}{2,4333} & 13 & 17 & 0 & عدد & \multirow{2}{*}{ دقياس المعايير المحاسبية الدولية } \\
\hline & & & 43.3 & 56.7 & 0 & $\%$ & \\
\hline \multirow{2}{*}{ ميتاز } & \multirow{2}{*}{0,49827} & \multirow{2}{*}{2,4000} & 12 & 18 & 0 & عدد & \multirow{2}{*}{ دقياس محاسبة مالية معمقة علاتي في امتحانات } \\
\hline & & & 40 & 60 & 0 & $\%$ & \\
\hline \multirow{2}{*}{ متاز } & \multirow[t]{2}{*}{0,50855} & \multirow[t]{2}{*}{2,5000} & 15 & 15 & 0 & عدد & \multirow{2}{*}{ نصفة عامة تمكني من المقاييس المحاسبية } \\
\hline & & & 50 & 50 & 0 & $\%$ & \\
\hline ضعيف & 0,67891 & 1,4333 & 3 & 7 & 20 & عدد & \\
\hline
\end{tabular}


إتقان اللغات الأجنبية كأسلوب لتحسين الاداء الأكاديمي لطلبة المحاسبة

\begin{tabular}{|c|c|c|c|c|c|c|c|}
\hline & & & 10 & 23.3 & 66.7 & $\%$ & مجال المحانية إعدادي لمقالات بحثية في باللغة الانجليزية \\
\hline \multirow{2}{*}{ ضعيف } & \multirow{2}{*}{0,62606} & \multirow[t]{2}{*}{1,4333} & 2 & 9 & 19 & عدد & \multirow{2}{*}{ إمكانية إعدادي لمقالات بحثية في المحاسبة باللغة الفرنسية } \\
\hline & & & 6.7 & 30 & 63.3 & $\%$ & \\
\hline \multirow{2}{*}{ متاز } & \multirow{2}{*}{0,61495} & \multirow{2}{*}{2,3667} & 13 & 15 & 2 & عدد & \multirow{2}{*}{ هند المؤسسات كانت ديامي بتقرير تربص ميداني فهمي في } \\
\hline & & & 43.3 & 50 & 6.7 & $\%$ & \\
\hline \multirow{2}{*}{ متاز } & \multirow[t]{2}{*}{0,61495} & \multirow[t]{2}{*}{2,3667} & 13 & 15 & 2 & عدد & \multirow{2}{*}{ في حساسبة أي مؤسسئ على اقتصادية فرصة عمل في } \\
\hline & & & 43.3 & 50 & 6.7 & $\%$ & \\
\hline \multirow[t]{2}{*}{ متوسط } & \multicolumn{2}{|c|}{2,1333} & \multicolumn{5}{|c|}{ المتوسط العام للمحوز } \\
\hline & \multicolumn{2}{|c|}{$\mathbf{0 , 3 6 5 5 3}$} & \multicolumn{5}{|c|}{ الانحراف المعياري للمحور } \\
\hline
\end{tabular}

المصدر: مخرجات برنامج SPSS

5- الإجابة على الأسئلة الفرعية

من خلال الجدول 3 الموضح أسفله نجد ما يلي: بالنسبة للسؤال الأول: لا توجد علاقة بين اتقان اللغات الأجنبية والتحصيل العلمي لطلبة المحاسبة بحيث أن مستوى المعنوية يزيد عن 5\% كما أن الارتباط بينهما ضعيف 31\%، ذلك ان اتقان اللغات الأجنبية يفسر \% من التحصيل العلمي للطبة المحاسبة، وكلما زاد اتقان اللغات الأجنبية بدرجة واحدة يرتفع التحصيل العلمي بمقدار 0.23 وحدة فقط. يمكن أن نفسر ذلك بالاعتماد على الواقع الحالي وهو أن أغلب الطلبة ينتهجون أسلوب التعلم السطحي(SLA) اختصار لـ " Surface Learning Approach"وهو نهج سلبي للتعلم اين يقوم فيه الطالب بتعلم ما هو مطلوب فقط(أي الحد الأدنى من المعلومات او ما قدم له فقط) لغرض اجتياز الاختبارات والحصول على العلامات فقط دون الخوض في التفاصيل، نظرا لأن الامتحانات الجامعية

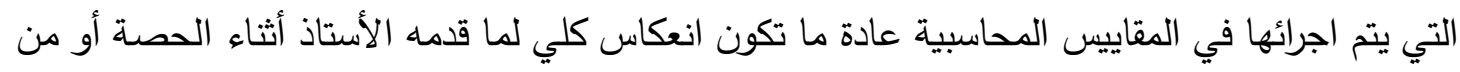
خلال مطبوعاته الجامعية أو كتبه الجامعية أو امتحاناته السابقة والتي تكون جميعها باللغة العربية خاصة مقياس المحاسبة المالية المعمقة، لذا لن يحتاج الطالب الى اتقان اللغات الأجنبية لقراءة وفهم الكتب لتبه

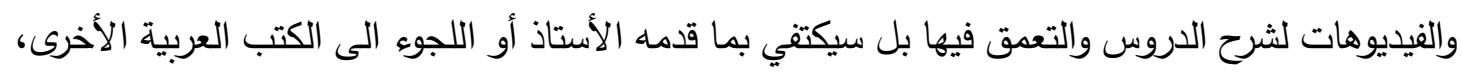
ولا ننسى عامل ضيق الوقت بالنسبة للرزنامة الجامعية التي تتميز بالاكتظاظ والتي تدفع الطالب الى انتهاج هذا الأسلوب وتجنب تعلم واستخدام اللغات الاجنبية.

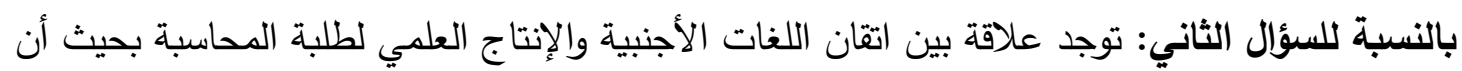

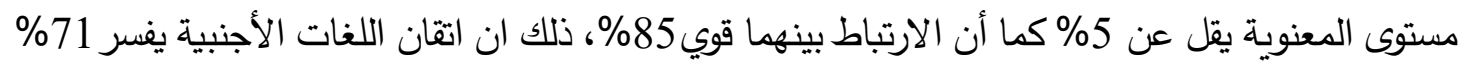
من الإنتاج العلمي للطلبة المحاسبة، وكلما زاد اتقان اللغات الأجنبية بدرجة واحدة يرتقع الإنتاج العلمي 
المجلا. 5، العدد. 1 (جوان 2021)؛ 565N: 2602-565

بمقدار 0.92 وحدة. يمكن أن نفسر ذلك بأن اتقان اللغات الأجنبية قادر على مساعدة طلبة المحاسبة في انتاج مقالات في مجلات دولية مصنفة وكذا يساعدهم في مطالعة الإنتاج العلمي الدولي، وهذا ما نشهده من خلال واقع طلبة المحاسبة عبر الوطن بحيث أن أغلبهم يقتصر نشرهم للأعمال العلمية على المجلات الوطنية الجزائرية فقط وأغلبها تكون باللغة العربية نظرا لافتقارهم للغات الأجنبية على عكس باقي التخصصات التي تدرس باللغات الأجنبية والتي يقوم طلبتها بالنشر دوليا. بالنسبة للسؤال الثالث: لا توجد علاقة بين اتقان اللغات الأجنبية وقدرة الاندماج المهني لطلبة المحاسبة

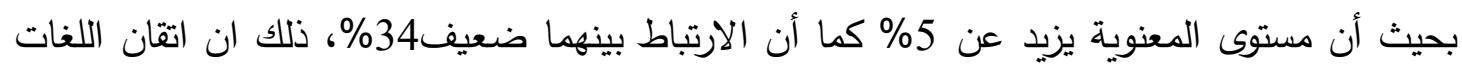
الأجنبية يفسر 11\% من الاندماج المهني للطبة المحاسبة، وكلما زاد اتقان اللغات الأجنبية بدرجة واحدة

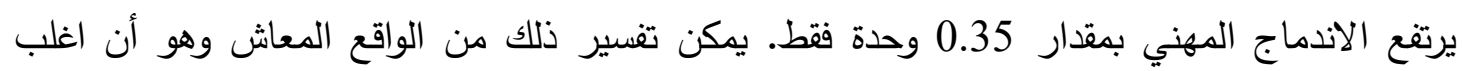
المؤسسات الجزائرية تخصص عاملين خاصين (أغلبهم ذوي خلفية أكاديمية أو انهم على اطلاع بالبرامج

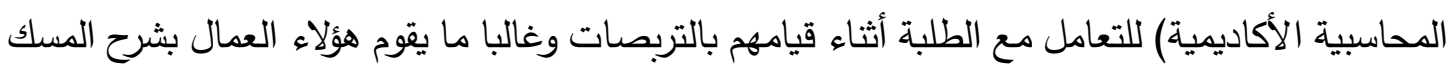

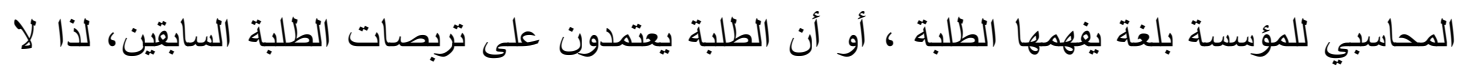
يحتاجون الى اتقان اللغات الأجنبية لتسهيل عملية اندماجهم وهو ما وضع شعور زائف لديهم بأن قدرتهم على الاندماج المهني في المستقبل ستكون كبيرة في حين ان غياب هؤلاء العمال أو أعمال الطالبة السابقين قد يصعب عليهم عملية الاندماج المهني. -بصفة عامة توجد علاقة بين اتقان اللغات الأجنبية والأداء الاكاديمي لطلبة المحاسبة بحيث أن مستوى علئ

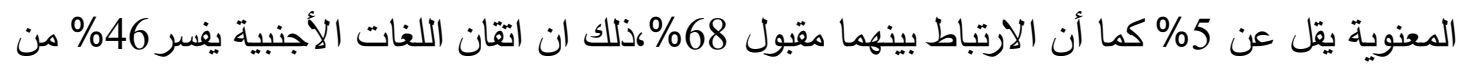
الأداء الاكاديمي للطلبة المحاسبة، وكلما زاد اتقان اللغات الأجنبية بدرجة واحدة يرتفع الأداء الاكاديمي بمقدار 0.46 وحدة.

جدول 3: الاختبارات الاحصائية للمتغيرات

\begin{tabular}{|c|c|c|c|c|c|}
\hline معادلة الانحدار & Sig F & $\mathbf{R}^{2}$ & $\mathbf{R}$ & المتغير التابع & المستقل \\
\hline$y 1=2.07+(0.23) X$ & 0.1000 & 0.09 & 0.31 & التحصيل العلمي(y1) & \multirow{4}{*}{ الأجنان اللغات } \\
\hline$y 2=0.06-(0.92) X$ & 0.0000 & 0.71 & 0.85 & الانتاج العلمي(y2) & \\
\hline $\mathrm{y} 3=1.80+(0.35) X$ & 0.069 & 0.11 & 0.34 & الاندماج المهني(y3) & \\
\hline$Y=1.36+(0.46) X$ & 0.0000 & 0.46 & 0.68 & الأداء الأكاديمي(Y) & \\
\hline
\end{tabular}

المصدر: مخرجات برنامج SPSS 
إتقان اللغات الأجنبية كأسلوب لتحسين الاداء الأكاديمي لطلبة المحاسبة

6- خاتمة

مما لا شك فيه يمثل المستوى الأكاديمي لطلبة المحاسبة أحد أهم أعمدة النهوض بالتعليم المحاسبي في الجزائر ، لذا من المهم الاهتمام بتحسين مستواهم باتباع أحدث الطرق والأساليب والمناهج العالمية، كالتركيز على تدريس مناهج المحاسبة بلغات أجنبية رائدة كالإنجليزية، كما هو سائد في أغلب دول العالمالم.

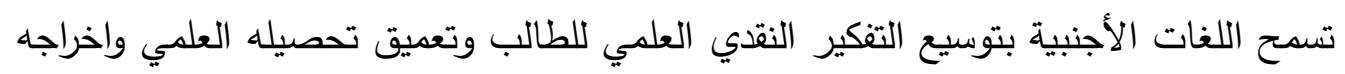
من حقل التعلم السطحي، مما يؤدي في نهاية المطاف الى الرفع من انتاجه العلمي وتحسين مستوى بكى اندماجه المهني مستقبلا ذلك أن اتقان اللغات الأجنبية -على الأقل لغرض المحاسبة-تساعد على الاطلاع على الإنتاج العلمي الدولي الحديث والرائد في مجال المحاسبة بدل الانغلاق على الإنتاج العلمي الجزائري فقط.

من خلال هذه الورقة البحثية توصلنا الى أن مستوى اتقان اللغات الأجنبية لدى طلبة المحاسبة

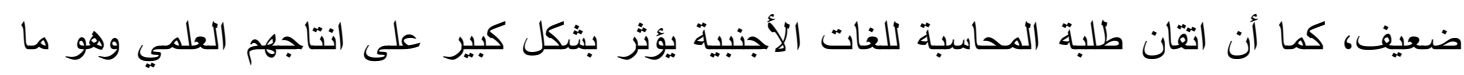

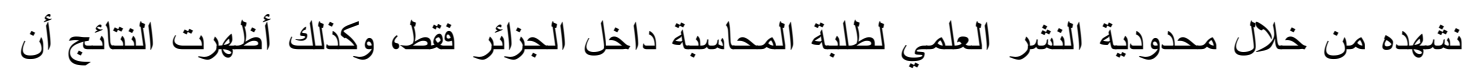
اتقان اللغات الأجنبية لا يؤثر على التحصيل العلمي والاندماج المهني المستقبلي لطلبة المحاسبة نظرا للأساليب السلبية التي تتبعها كل من البيئة الاكاديمية والبيئة المهنية الجزائرية بحيث نشهد انه من خلال واقع الحال يقتصر التعليم المحاسبي الأكاديمي على اللغة العربية وعلى معطيات الأساتذة فقط بعيدا عن ون الممارسات المهنية المحلية والدولية، في حين أن البيئة المهنية تهمل احتياجات طلبة المحاسبة من خلال سياسة ملأ الفراغ التي تتبع في تربصات الميدانية.

نوصي من خلال هذه الدراسة بضرورة مراجعة أساليب التعليم المحاسبي في الجزائر عن طريق تُبات تغيير لغة المناهج الى لغات دولية وتقريبها من الممارسات المهنية المحلية والدولية، وكذا مراجعة القوانين التي تربط المؤسسات الاقتصادية بالمؤسسات الجامعية وجعلها أكثر ملائمة لطلبة المحاسبة.

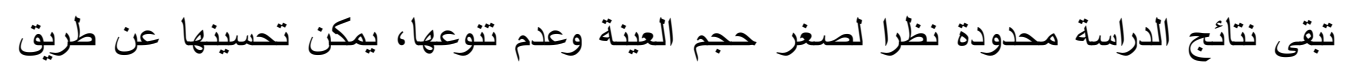
التتويع في مستويات الطلبة المستجوبين وكذا توسيع الجامعات محل الدراسة، وتحسين طرق قياس متغيرات الدراسة ومقارنة النتائج بتجارب عالمية. 
المجلد. 5، العدد. 1 (جوان 2021)؛ 5655- 2602-

\section{المراجع}

أمعرف سعاد عياشن كبلان عبدالسلام علي. (2009). ليبيا رقم براءة الاختراع 866887. بن ونيسة ليلى،بن عبو جيلال. (2015). واقع جودة التعليم العالي في الجزائر من منظور التصنيفات الدولية، مجلة الدراسات الاقتصادية الكمية، 1(1)، 107-117.

فاريح محمد. (ديسمبر , 2019). تعليم اللغة العربية لأغراض أكادميية في برنامج الكثف. المجلة العربية الدولية للتربية والتعليم، 03(02)، 15-24. قادري حليمة. (2009).

قاصدي فايزة، طبيب فتيحة. (2017). مفهوم الجودة في التعليم العالي. مجلة جيل العلوم الإنسانية والاجتماعية، $.171 ، 27$

محمد ابوالقاسم زكري. (2013). التحديات والمشاكل المعاصرة التي تواجه الجودة الشاملة للتعليم المحاسبي في ليبيا (دراسة حالة في كلية الاقتصاد فرع الجفارة). المؤتمر السنوي الخامس للمنظمة العربية لضمان الجودة في التعليم حول نظم الجودة وتطبيقها باستخدام أساليب تكنولوجية مبتكرة لضمان الجودة في التعليم. تونس.

محمود إسماعيل صالح. (27 ديسمبر, 2013). تعليم العربية لأغراض خاصة . تاريخ الاسترداد 22 06, 2021، من مدونة الدكتور محمود إسماعيل صالح: http://dr-mahmoud-ismail-saleh.blogspot.com

Abaidoo, A. (2018). Factors contributing to academic performance of students in a Junior High School. Consulté le 06 23, 2021, sur GRIN: https://www.grin.com. Abdirahman M. Addow, A. H. (2013). ENGLISH LANGUAGE PROFICIENCY AND ACADEMIC ACHIEVEMENT FOR UNDERGRADUATE STUDENTS IN SOMALIA. Educational Research International, 2(2).

Agustina, T. (2014, 05). ENGLISH FOR SPECIFIC PURPOSES (ESP): AN APPROACH OF . Beta, 07(01), 37-63.

Ahmed Abubakar, H. H. (2018). New Tools for Measuring Global Academic Performance. SAGE Open, 1-10.

Aysha Khaled Almajed, A. H. (2015, June). ENGLISH LANGUAGE AND ACCOUNTING EDUCATION. European Journal of Accounting Auditing and Finance Research, 3(6), 12-25.

كلية العلوم الاقتصادصة و العلوم التجارية و علوم التسيير جامعة أم البو اقي 
رجاء دويس وفاتح أحمية

إتقان اللغات الأجنبية كأسلوب لتحسين الاداء الأكاديمي لطلبة المحاسبة

ferrari, g. p. (2016, 09 29). Qu'est-ce que le Français sur Objectifs Specifiques?

Consulté le 06 23, 2021, sur FOS pour toi: https://fospourtoi.wordpress.com.

Harsch, C. (2017, April 1). Proficiency. ELT Journal, 71(2), 250-253.

Lamas, H. A. (2015). School Performance. Propósitos y Representaciones, 3(1), 313 386.

M S Sumbawati, Y. A. (2018). The impact of research-based learning on student's academic performance and motivation. IOP Conference Series: Materials Science and Engineering.

Nascimento, A. A. (2005, 09).

prondzynski, F. v. (s.d.). What is quality in higher education? Consulté le 06 26, 2021, sur A university Blog: https://universitydiary.wordpress.com.

Saumya Kumar, M. A. (2021). Defining And Measuring Academic Performance of Hei Students-A Critical Review . Turkish Journal of Computer and Mathematics Education, 12(6), 3091-3105.

Tachibana, C. (2017, 2 10). New tools for measuring academic performance. Consulté le 07 21, 2021, sur science: https://www.sciencemag.org.

Trace, J. H. (2015). An overview of language for specific purposes. 1-23. Honolulu: University of Hawai'i. 


\title{
MASTERING FOREIGN LANGUAGES AS A METHOD TO IMPROVE THE ACADEMIC PERFORMANCE OF ACCOUNTING STUDENTS: EVIDENCE FROM UNIVERSITY OF JIJEL Raja DOUIS $^{\text {a }}{ }^{\star}$ Fateh AHMIA $^{\mathrm{b}}$ \\ a.rajadouis@gmail.com, university of Jijel, Algeria \\ a.Fateh742002@yahoo.fr, university of Jijel, Algeria
}

Received date: 30/ 05/20**, Accepted date: 20/ 06/2021, online publication date: 30/ 06/2021

\begin{abstract}
This study seek to highlight the impact of foreign languages proficiency on academic performance of accounting students, in the context of finding methods that would improve Algeria's accounting education, to obtain the study results the descriptive, analytical methods have been followed, and survey have been distributed on 30 student male/female from accounting field in Jijel university. We came to that languages proficiency significantly improve scientific production of accounting students while it does not affect their learning and professional integration in Algerian environment.
\end{abstract}

Keyword: foreign languages; academic performance; accounting education; scientific production.

JEL Code: M41; Z13 ; A29.

\footnotetext{
^ Corresponding Author
}

كلية العلوم الاقتصادصة و العلوم التجارية و علوم التسبير 